UC-414

Issued: January 1994

B-jet Tagging Using the GEM Central Tracker

M. L. Brooks 


\title{
B-JET TAGGING \\ USING THE GEM CENTRAL TRACKER
}

by

M. L. Brooks

\begin{abstract}
The efficiency for tagging $b$-jets with the full GEM central tracker has been determined using a GEANT simulation to be approximately $35 \%$ for b-jets with $\mathrm{p}_{\mathrm{T}}>50 \mathrm{GeV} / \mathrm{c}$ while the fraction of other quark jets that are misidentified as b-jets is on the order of $1-3 \%$.
\end{abstract}

\section{Introduction}

The efficiency for tagging b-jets with the full GEM central tracker has been studied, using a GEANT simulation of the tracker which included full geometry and material simulation, full detector response simulation in the silicon portion of the tracker, and full pattern recognition to find tracks within the $b$-jets. JETSET was used to generate the jet events.

\section{Method and Results}

To determine the efficiency for tagging b-jets, jets were generated with various $\mathrm{p}_{\mathrm{T}}$ values with the JETSET routine LU1ENT, the full pattern reconstruction code was run on the tracks generated, and the impact parameter of selected tracks was examined. The tracks that were selected from the b-jet tracks had to pass the following criteria:

1) Track found with pattern recognition

2) $\mathrm{p}_{\mathrm{T}} \geq 0.8 \mathrm{GeV} / \mathrm{c}$

3) $\mathrm{R} \leq 0.5$ (track within cone of jet) jet origin.

4) Impact parameter has correct sign, consistent with a track originating at the

The $0.8 \mathrm{GeV} / \mathrm{c}$ cutoff was used because the pattern recognition efficiency for finding tracks drops sharply below about $1 \mathrm{GeV} / \mathrm{c}$ and the impact parameter resolution becomes large compared to the average impact parameter of a b-jet track below $0.8 \mathrm{GeV} / \mathrm{c}$. The cone cut and the cut on the correct sign of the impact parameter helped reduce the number of tracks counted with poorly measured track parameters.

The jet was then tagged as a b-jet if three or more tracks that pass the above criteria had an impart parameter greater than $3^{*} \sigma_{b}$, where $\sigma_{b}$ is the parameterized sigma of the impact parameter resolution of the GEM central tracker, and is given by:

$$
\sigma_{\mathrm{b}}=\left[24^{2}+\left(200 / \mathrm{p}_{\mathrm{T}}\right)^{2}+(300 * \cos (\theta) / \mathrm{pT})^{2}\right]^{1 / 2} \text { microns }
$$


where $\mathrm{pT}_{\mathrm{T}}$ is the perpendicular momentum of the track, and $\theta$ is the angle of the track relative to the $\mathrm{z}$ axis.

The efficiency for finding all of the tracks in a b-jet with $\mathrm{pT}_{\mathrm{T}} \geq 0.8 \mathrm{GeV} / \mathrm{c}$ varied from 75 $95 \%$ with $\mathrm{pT}$ of the jet $=30-200 \mathrm{GeV} / \mathrm{c}$, as can be seen in Figure 1 .

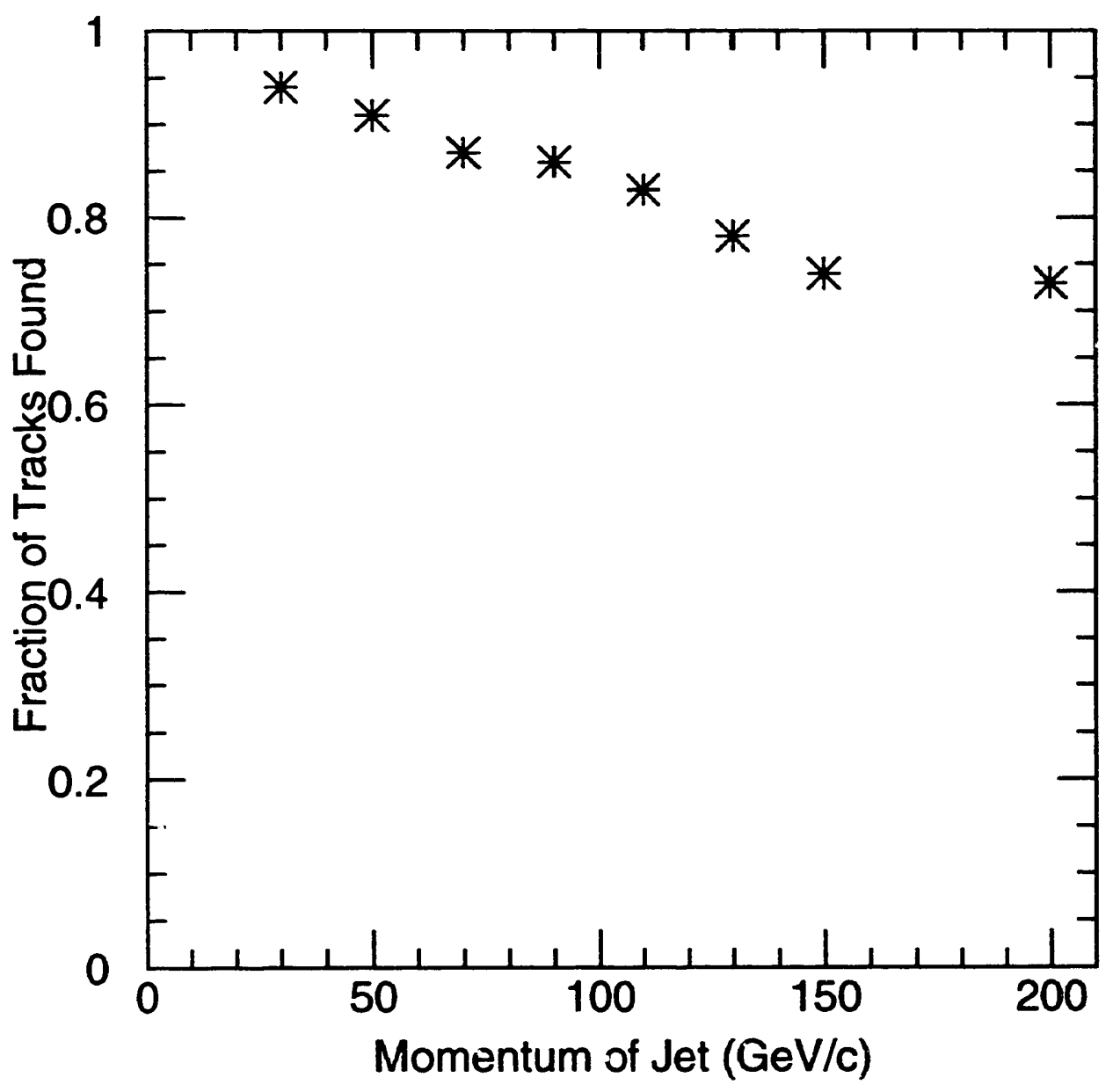

Figure 1. The fraction of all tracks above $0.8 \mathrm{GeV} / \mathrm{c}$ that were found with the pattern recognition vs. the pT of the jet.

JETSET was used to generate b-jets with various $\mathrm{pT}$, distributed over the entire $\phi$ and $\eta$ range of the tracker, as vell as $\mathrm{u}-, \mathrm{d},-\mathrm{s},-$ and $\mathrm{c}$-jets. The impact parameter distribution of the tracks from $\mathrm{b}-, \mathrm{c}-$, and $\mathrm{u}$ - jets with $\mathrm{pT}$ of $90 \mathrm{GeV} / \mathrm{c}$ can be seen in Figure 2. The energy distribution of the tracks that pass through four or more layers of the tracker coming from the bjets can be seen in Figure 3. These two figures indicate the difference in impact parameter distributions among the different types of jets that was taken advantage of to tag the b-jets, as well as the relatively low energy distributions of the tracks emerging from the jets, which makes the tagging difficult both from a pattern recognition standpoint as well as because the impact parameter resolution degrades significantly at low momentum. 


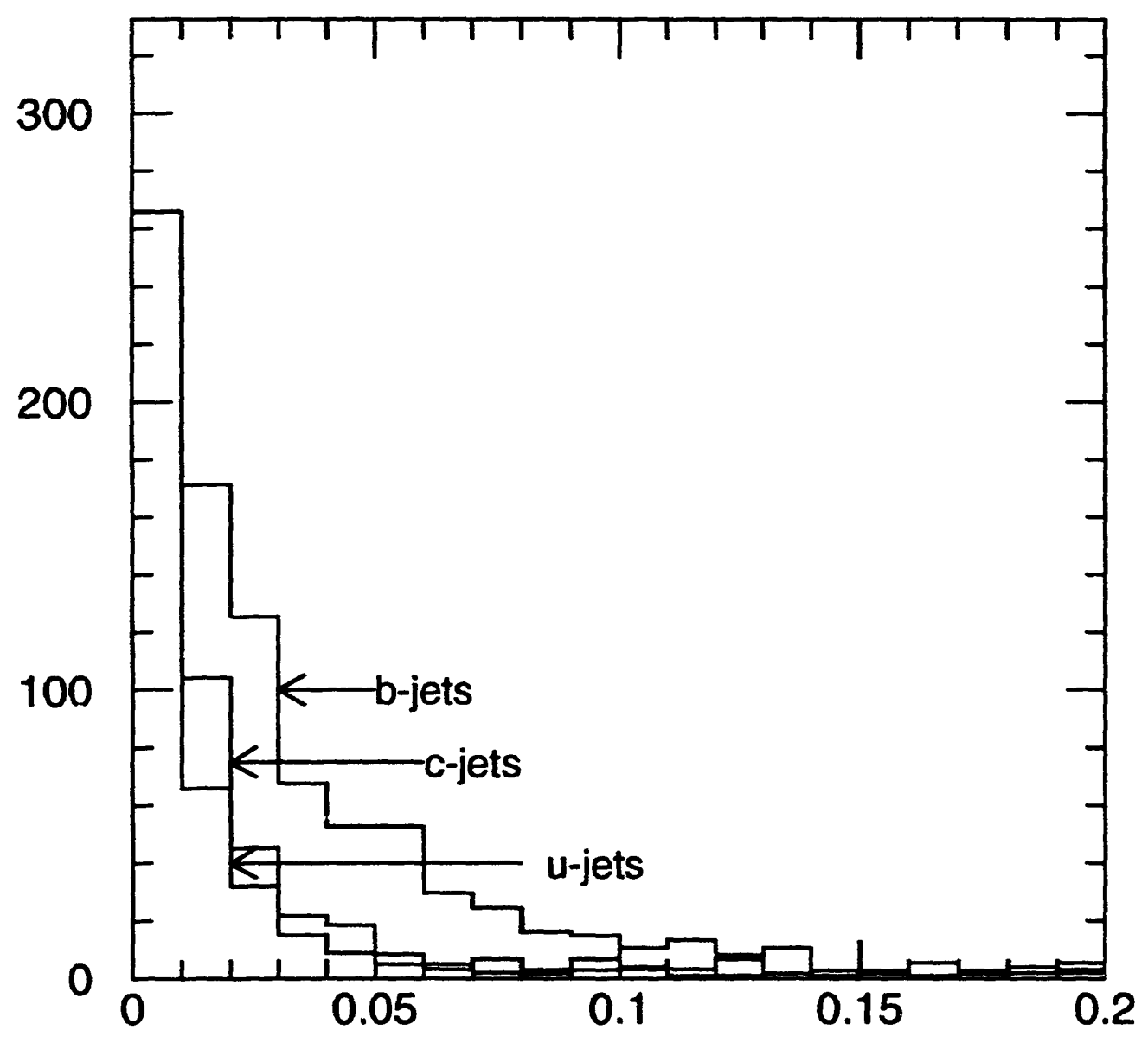

Figure 2. The impact parameter distribution of all tracks above $0.8 \mathrm{GeV} / \mathrm{c}$ from $90 \mathrm{GeV} / \mathrm{c} \mathrm{PT}$ b-, c-, and ujets. 


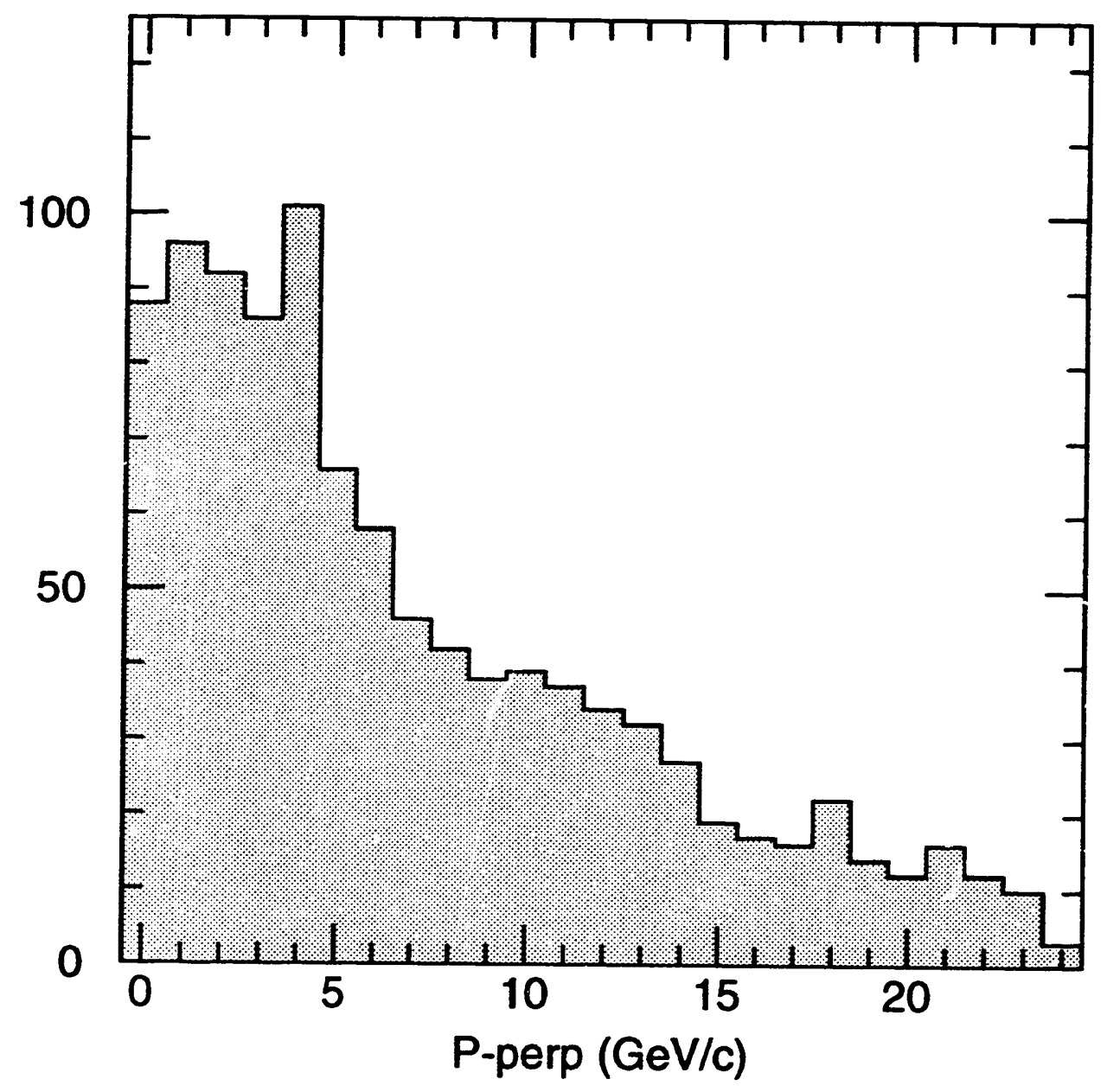

Figure 3. The energy distribution of all tracks from $90 \mathrm{GeV} / \mathrm{c}$ PT b-jets that pass through four or more layers of the central tracker.

The efficiency for correctly tagging b-jets vs. the pT of the jet is shown in Figure 4 along with the average efficiency for incorrectly tagging $u-, d-, s_{-}$, and $c$-jets as $b$-jets. The $b$-jet tagging efficiency varies from $12 \%$ (at $30 \mathrm{GeV} / \mathrm{c} \mathrm{PT}$ ) to approximately $35 \%$ at high pT. At the low momenta, the tagging efficiency decreases mostly because the impact parameter resolution of low momenta particles is large, causing the three sigma cut to reject most of the b-jet tracks. 


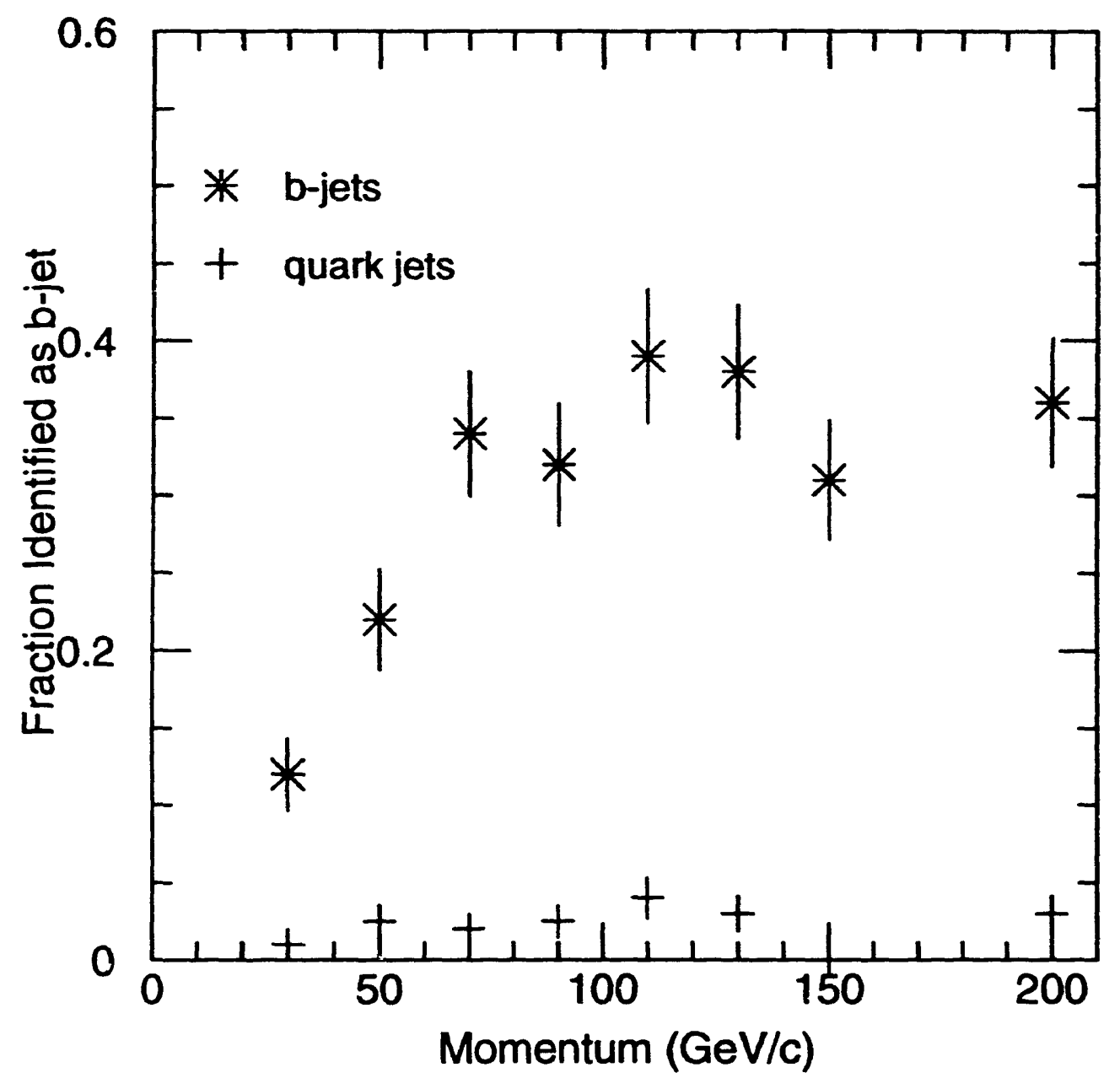

Figure 4. The fraction of b-jets that are correctly tagged as b-jets and the average fraction of $u-, d-, 5-$, and $c-$ jets that are incorrectly tagged as b-jets vs. the pT of the jet.

\section{Conclusion}

The GEM central tracker is capable of correctly tagging b-jets with pT $=(70,200) \mathrm{GeV} / \mathrm{c}$ at the $35 \%$ level, while still rejecting most other quark jets, by counting the number of tracks with impact parameters that are large relative to the impact parameter measurement error. 

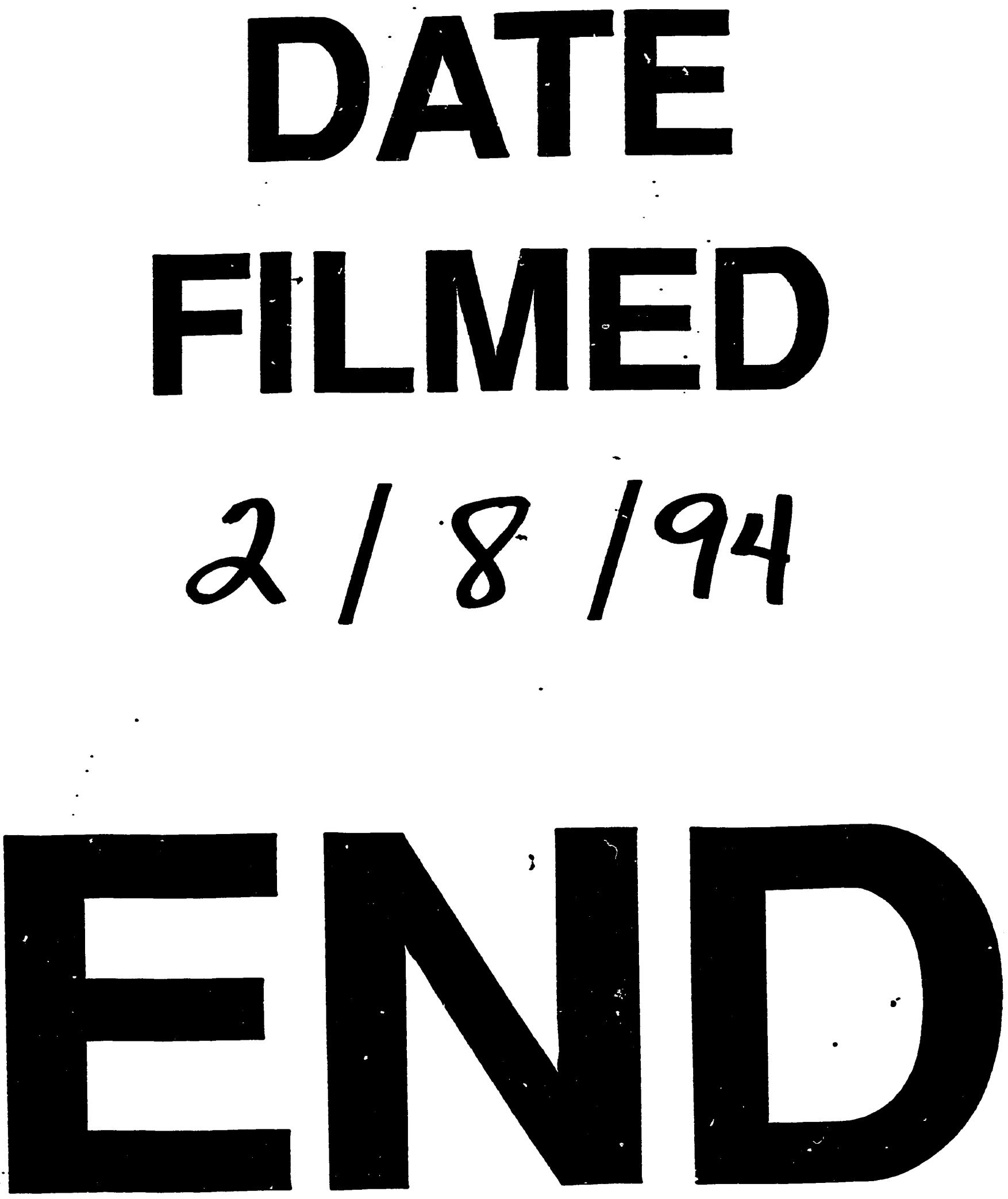